\title{
Addendum: Recognition of RNA virus by RIG-I results in activation of CARD9 and inflammasome signaling for interleukin $1 \beta$ production
}

Hendrik Poeck, Michael Bscheider, Olaf Gross, Katrin Finger, Susanne Roth, Manuele Rebsamen, Nicole Hannesschläger, Martin Schlee, Simon Rothenfusser, Winfried Barchet, Hiroki Kato, Shizuo Akira, Satoshi Inoue, Stefan Endres, Christian Peschel, Gunther Hartmann, Veit Hornung \& Jürgen Ruland

Nat. Immunol. 11, 63-69 (2010); published online 15 November 2009; addendum published after print 15 May 2013

In 2010 we reported in Nature Immunology how the sensing of cytosolic RNA triggers the generation of mature interleukin $1 \beta$ (IL-1 $\beta$; Poeck et al., Nat. Immunol. 11, 63-69 (2010)). We demonstrated that ligation of the RNA helicase RIG-I triggered the adaptor CARD9 downstream of the signaling adaptor MAVS (IPS-1) to induce the transcription factor NF- $\kappa \mathrm{B}$ for the generation of pro-IL- $1 \beta$ and in parallel activated caspase- 1 for the generation of mature IL- $1 \beta$. We presented data that indicated similar generation of pro-IL-1 $\beta$ and IL- $1 \beta$ in wild-type bone marrow-derived dendritic cells (BMDCs) and BMDCs deficient in CARD9 or the adaptor Bcl-10 after transfection of the synthetic DNA poly(dA:dT) (Fig. 3c (right) and Fig. 4c,e,f). However, additional experiments in our laboratory have shown that CARD9- or Bcl-10-deficient BMDCs have impaired production of pro-IL- $1 \beta$ and IL- $1 \beta$ not only after sensing of RNA but also after the transfection of poly(dA:dT). We speculate that a particular aliquot of poly(dA:dT) used during the preparation of our study published in 2010 might have been contaminated with lipopolysaccharide or some other CARD9-independent trigger of the innate immune system. Still, the central conclusions of that manuscript (that RIG-I signals via CARD9 and the inflammasome to control IL-1 $\beta$ ) remain unchanged and have been confirmed by multiple independent laboratories. Nevertheless, we would like to correct the idea that CARD9- or Bcl-10-deficient cells have normal IL-1 $\beta$ responses to the transfection of poly(dA:dT).

\section{Corrigendum: Aiolos promotes $\mathrm{T}_{\mathrm{H}} 17$ differentiation by directly silencing $/ / 2$ expression}

Francisco J Quintana, Hulin Jin, Evan J Burns, Meghan Nadeau, Ada Yeste, Deepak Kumar, Manu Rangachari, Chen Zhu, Sheng Xiao, John Seavitt, Katia Georgopoulos \& Vijay K Kuchroo

Nat. Immunol. 13, 770-777 (2012); published online 1 July 2012; corrected after print 21 September 2012

In the version of this article initially published, some data in the bottom right plot in Figure $3 \mathrm{~d}$ were partially obscured. The error has been corrected in the HTML and PDF versions of the article.

\section{Corrigendum: Control of amino-acid transport by antigen receptors coordinates the metabolic reprogramming essential for $T$ cell differentiation}

\author{
Linda V Sinclair, Julia Rolf, Elizabeth Emslie, Yun-Bo Shi, Peter M Taylor \& Doreen A Cantrell \\ Nat. Immunol. 14, 500-508 (2013); published online 24 March 2013; corrected online 25 June 2013
}

In the version of this article initially published, the legend for Figure $7 \mathrm{~b}$ incorrectly included mutant cells. The correct legend should read “...OT-I lymph node T cells...." The error has been corrected in the HTML and PDF versions of the article.

\section{Corrigendum: $T_{H} 2$, allergy and group 2 innate lymphoid cells}

Paula Licona-Limón, Lark Kyun Kim, Noah W Palm \& Richard A Flavell

Nat. Immunol. 14, 536-542 (2013); published online 20 May 2013; corrected after print 25 June 2013

In the version of this article initially published, an arrow was incorrectly included between the MPP2 cell and CD4 ${ }^{+}$cell in the top row of Figure 2. The correct figure has no arrow there. The error has been corrected in the HTML and PDF versions of the article.

\section{Erratum: Research Highlight: Latent enhancement}

\section{Zoltan Fehervari}

Nat. Immunol. 14, 204 (2013); published online 15 February 2013; corrected after print 12 July 2013

In the version of this article initially published, the year of the citation is incorrect. The correct year is 2013 . The error has been corrected in the HTML and PDF versions of the article. 